\title{
SYNTHESIS OF NEW PIPERAZINYL INDOLYL PROPANONES AND PRELIMINARY CNS PHARMACOLOGICAL EVALUATION IN MICE
}

\author{
A.R. Subramanian, S.A.V. Raghavan, R.J. Babu, C.N.V.H.B. Gupta, N. Sridhar, \\ A. Veeranjaneyulu, the LATE P. Parimoo and P. Srinivas \\ Department of Pharmacy, Birla Institute of Technology \& Science, Pilani-333 031, India
}

\begin{abstract}
In a continuing progamme to discover antipsychotic agents with a reduced propensity toward extrapyramidal side effects, the synthesis of a series of novel piperazinyl indolyl propanones by a mannich reaction is described. These compounds were evaluated for their dopaminergic $\left(\mathrm{D}_{1} / \mathrm{D}_{2}\right)$ and serotonergic $\left(5-\mathrm{HT}_{2}\right)$ receptor antagonism in a preliminary in vivo pharmacological evaluation in mice and have shown antagonism to the dopaminergic $\left(\mathrm{D}_{1} / \mathrm{D}_{2}\right)$ receptor, but failed to antagonize the serotonergic $\left(5-\mathrm{HT}_{2}\right)$ receptor to a significant extent.
\end{abstract}

\section{Introduction}

Appropriately substituted indoles have been of interest to the medicinal chemist especially because of the widespread occurrence of the indole nucleus among both naturally occurring and synthetic psychoactive compounds (1). As a result of the large number of indole derivatives that are psychoactive, it was of interest to investigate the effect of bringing in suitable pharmacophores onto an indolyl system for effective CNS function.

Numerous workers have implicated indolylalkylamines as having an important role in CNS function (2). The various structural features of indolylalkylamines which are to be considered in order to bring about structural modifications for effective CNS function are (a) the terminal amine function, $(b)$ side-chain, and (c) the indole-1 position (3).

Of these, the terminal amine group of an indolylalkylamine interacts electrostatically with various receptors and the steric bulk of the amine plays an important role in determining the affinity. Maintaining the length of the side-chain within four methylene groups and the presence of an alkyl or an aryl/substituted aryl moiety at the indole-1 position have been reported to show varying affinities, depending on the presence of other substituents on the parent indole system (4).

In the present work, we have chosen arylpiperazines, owing to their steric bulk, as the terminal amine function, a 4-fluorophenyl moiety as the indole-1 substituent and maintaining a chain length of three methylene units between the indole system and the terminal amine function. The chemical starting point chosen for this effort was 3-acetyl indole, which is reported to be a structural template for certain CNS activities (5). 
Using 3-acetyl indole as the substrate we have recently reported the first synthesis of 1-(4-fluorophenyl)-3-acetyl indole 1 by an analogous Ullmann condensation procedure involving 3-acetyl indole and 4-fluoroiodobenzene in the presence of a suitable catalyst (6). The active methylene of $\underline{1}$ would be available for further modifications by bringing in suitable pharmacophores to generate potential agents for prospective CNS activities. The Mannich reaction which is a classic three-component system in which "hydrogen active" substrates react with putative imine species that arise from condensation of an amine with an aldehyde (7), offers the best solution of bringing in a terminal amine function and a methylene onto $\underline{1}$.

In this communication we report the synthesis and preliminary pharmacological evaluation of arylpiperazinyl mannich bases of 1-(4-fluorophenyl)-3-acetyl indole as Novel Piperazinyl Indolyl Propanones for prospective CNS activities.

\section{Experimental}

All melting points were determined on a Buchi model 530 melting point apparatus and are uncorrected. Infrared spectra were recorded on JASCO IR Report-100 spectrophotometer and are given in $\mathrm{cm},{ }^{-1}$ recorded as a mull, unless specified. The 'H NMR spectra, were recorded using Bruker AC 300F NMR spectrometer or Jeol GSX 400 NMR spectrometer and are reported in $\delta$ units (ppm) relative to tetramethylsilane as internal standard. Elemental analyses are indicated by the symbols of the elements and were within $\pm 0.4 \%$ of the theoretical values.

General Procedure for the synthesis of Arylpiperazinyl Mannich Bases of 1-(4fluorophenyl)-3-acetyl indole

Literature procedure (10) was used as the basis for the synthesis of products $\underline{\mathbf{2}} \mathbf{a}-\mathbf{j}$. In a 50 $\mathrm{ml}$ round bottom flask equipped with a reflux condenser and $\mathrm{N}_{2}$ inlet were placed 0.0024 mol of 1-(4-fluorophenyl)-3-acetyl indole $1,0.0036 \mathrm{~mol}$ of paraformaldehyde, 0.0024 mol of the N-4 unsubstituted arylpiperazine and $15 \mathrm{ml}$ of absolute ethanol. The $\mathrm{pH}$ was adjusted to 4 and the mixture was refluxed for 29 hours. The solvent was removed under reduced pressure to give the product as the hydrochloride salt of the mannich base. Compounds whose salts were unstable were converted into their fumarates or isolated as their free bases.
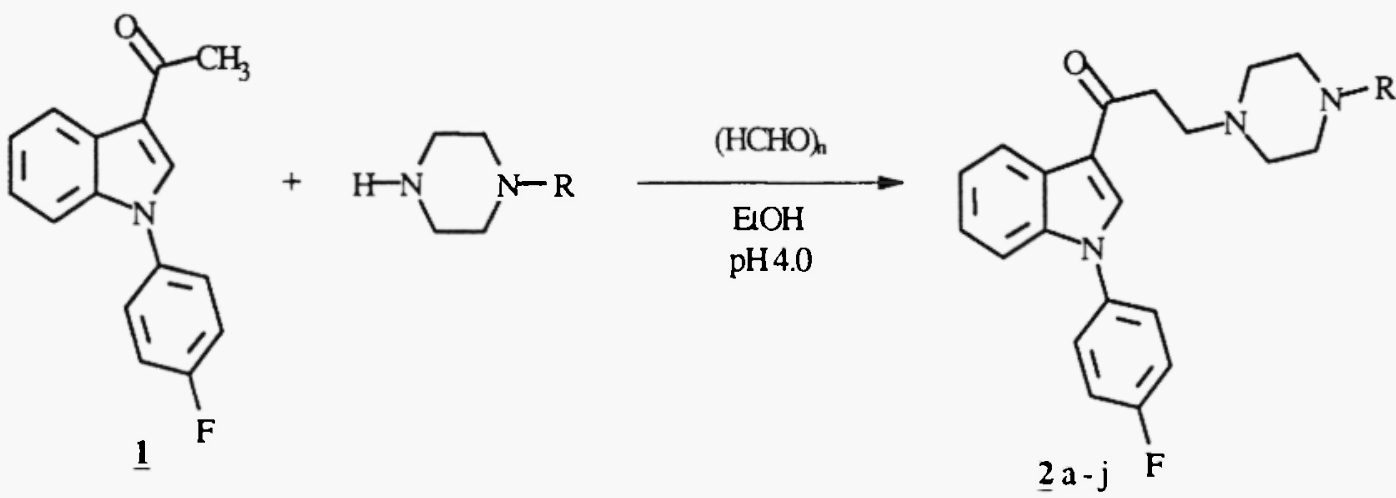
1-[1-(4-Fluorophenyl)-1H-3-indolyl]-3-(4-phenyl piperazin-1-yl)-1-propanone $\underline{2 a}$

mp. $178^{\circ} \mathrm{C}$; Yield 52\% (isopropyl alcohol); IR (cm ): 1640 (C=O); 1595 (aromatic); 1575 (carboxylate); 1160 (C-N). ${ }^{1} \mathrm{H}$ NMR (DMSO-d 6 ): 7.28-6.46 (m,14H,Ar-H); 3.76-3.12 (m,10H, CHz); $2.51\left(\mathrm{~s}, 2 \mathrm{H}, \mathrm{CH}_{2}\right)$. Anal. $\mathrm{C}_{27} \mathrm{H}_{26} \mathrm{~N}_{3} \mathrm{OF} . \mathrm{C}_{4} \mathrm{H}_{4} \mathrm{O}_{4}$

3-[4-(3-Chlorophenyl)piperazin-1-yl]-1-[1-(4-fluorophenyl)-1H-3-indolyl]-1-propanone $\underline{2 b}$

mp $145^{\circ} \mathrm{C}$; Yield 47\% (methanol-ether); IR ( $\left.\mathrm{cm}^{11}\right): 2495$ ( ${ }^{+} \mathrm{NHCl}$ ); $16+0(\mathrm{C}=\mathrm{O}) ; 1595$ (aromatic); 1550,1180 (C-N) . ${ }^{1} \mathrm{H}$ NMR (DMSO-d 6$): 8.30-6.73$ (m,13H,Ar-H); 3.76-2.84(m,10H, CH $\left(\mathrm{s}, 2 \mathrm{H}, \mathrm{CH}_{2}\right)$. Anal. $\mathrm{C}_{27} \mathrm{H}_{25} \mathrm{~N}_{3} \mathrm{OFCl}$. $2 \mathrm{HC}$

3-[4-(4-Chlorophenyl)piperazin-1-yl]-1-[1-(4-fluorophenyl)-1H-3-indolyl]-1-propanone 2 c

mp $210^{\circ} \mathrm{C}$; Yield 51\% (methanol-ether); IR $\left(\mathrm{cm}^{-1}\right): 2495\left({ }^{+} \mathrm{NHCl}\right) ; 1640(\mathrm{C}=\mathrm{O}) ; 1595$ (aromatic); 1540,1180 (C-N). ${ }^{1} \mathrm{H}$ NMR (DMSO-d $\left.\mathrm{d}_{6}\right): 8.29-6.57$ (m,13H,Ar-H); 3.99-2.90(m,10H, CH (s,2 $\mathrm{H}, \mathrm{CH}_{2}$ ). Anal. $\mathrm{C}_{27} \mathrm{H}_{25} \mathrm{~N}_{3} \mathrm{OFCl} .2 \mathrm{HC}$.

1-[1-(4-Fluorophenyl)-1H-3-indolyl]-3-(4-(4-fluorophenyl)piperazin-1-yl)-1-propanone $\underline{2 d}$

$\mathrm{mp} 175^{\circ} \mathrm{C}$; Yield $49 \%$ (methanol-ether); IR $\left(\mathrm{cm}^{-1}\right): 2475(+\mathrm{NHCl}), 1640(\mathrm{C}=\mathrm{O}) ; 1605$ (aromatic); 1520, 1180 (C-N). 'H NMR (DMSO-d $\left.\mathrm{d}_{6}\right): 8.32-7.05$ (m,13H,Ar-H); 3.77-3.16(m,10H, $\mathrm{CH}_{2}$ ); 2.52 (s,2H, $\mathrm{CH}_{2}$ ). Anal. $\mathrm{C}_{27} \mathrm{H}_{25} \mathrm{~N}_{3} \mathrm{OF}, 2 \mathrm{HCl}$

1-[1-(4-Fluorophenyl)-1H-3-indolyl]-3-[4-(2-methoxyphenyl)piperazin-1-yl]-1-propanone $\underline{2 e}$

$\mathrm{mp} 135^{\circ} \mathrm{C}$; Yield 46\% (methanol-ether); IR ( $\left.\mathrm{cm}^{-1}\right): 2475\left({ }^{+} \mathrm{NHCl}\right) ; 1650(\mathrm{C}=\mathrm{O}) ; 1595$ (aromatic); 1530,1180 (C-N); 1235 (Ar-O-CH3). ${ }^{1} \mathrm{H}$ NMR (DMSO-d $\left.\mathrm{d}_{6}\right): 8.32-6.93$ (m,13H,Ar-H), $3.80-$ $3.31\left(\mathrm{~m}, 10 \mathrm{H}, \mathrm{CH}_{2}\right) ; 3.20\left(\mathrm{~s}, 3 \mathrm{H}, \mathrm{Ar}-\mathrm{O}-\mathrm{CH}_{3}\right) ; 2.52\left(\mathrm{~s}, 2 \mathrm{H}, \mathrm{CH}_{2}\right)$. Anal. $\mathrm{C}_{28} \mathrm{H}_{28} \mathrm{~N}_{3} \mathrm{O}_{2} \mathrm{~F} .2 \mathrm{HCl}$

1-[1-(4-Fluorophenyl)-1H-3-indolyl]-3-[4-(3-methoxyphenyl)piperazin-1-yl]-1-propanone $2 f$ $\mathrm{mp} 90^{\circ} \mathrm{C}$; Yield $49 \%$ (ethanol-petroleum ether); IR (cm.1): 1660(C=O); 1600 (aromatic); 1520, 1180 (C-N); 1260 (Ar-O-CH $)_{3}$. 'H NMR (DMSO-dos): 8.47-6.38 (m,13H,Ar-H); 3.99-3.49(m,10H,CH ${ }_{2}$ ); 3.07 (s,3H,Ar-O- $\left.\mathrm{CH}_{3}\right) ; 2.58\left(\mathrm{~s}, 2 \mathrm{H}, \mathrm{CH}_{2}\right)$. Anal. $\mathrm{C}_{28} \mathrm{H}_{28} \mathrm{~N}_{3} \mathrm{O}_{2} \mathrm{~F}$

1-[1-(4-Fluorophenyl)-1H-3-indolyl]-3-[4-(4-methoxyphenyl)piperazin-1-yl]-1-propanone $2 \mathrm{~g}$

$\mathrm{mp} 151^{\circ} \mathrm{C}$; Yield 54\% (methanol-ether); IR (cm '): 2350 ( $\left.+\mathrm{NHCl}\right) ; 1645(\mathrm{C}=\mathrm{O}) ; 1600$ (aromatic); 1510,1180 (C-N); 1210 (Ar-O-CH3). ${ }^{1} \mathrm{H}$ NMR (DMSO-d 6 ): $8.31-6.83$ (m,13H,Ar-H); $3.69-$ $3.36\left(\mathrm{~m}, 10 \mathrm{H}, \mathrm{CH}_{2}\right) ; 3.22\left(\mathrm{~s}, 3 \mathrm{H}, \mathrm{Ar}-\mathrm{O}-\mathrm{CH}_{3}\right) ; 2.52\left(\mathrm{~s}, 2 \mathrm{H}, \mathrm{CH}_{2}\right)$. Anal. $\mathrm{C}_{28} \mathrm{H}_{28} \mathrm{~N}_{3} \mathrm{O}_{2} \mathrm{~F} .2 \mathrm{HCl}$

I-[1-(4-Fluorophenyl)-1H-3-indolyl]-3-[4-(3-trifluoromethyl phenyl) piperazin-1-yl]-1-propan one $\underline{2 h}$

mp $220^{\circ} \mathrm{C}$; Yield 59\% (methanol-ether); IR $\left(\mathrm{cm}^{-1}\right): 2525\left({ }^{+} \mathrm{NHCl}\right) ; 1650(\mathrm{C}=\mathrm{O}) ; 1590$ (aromatic); $1540,1175(\mathrm{C}-\mathrm{N}) ; 1120\left(\mathrm{CF}_{3}\right) \cdot{ }_{1}^{1} \mathrm{H}$ NMR (DMSO-d 6$): 8.33-7.10$ (m,13H,Ar-H); 3.99-3.03(m,10H,CH $2.51\left(\mathrm{~s}, 2 \mathrm{H}, \mathrm{CH}_{2}\right)$. Anal. $\mathrm{C}_{28} \mathrm{H}_{25} \mathrm{~N}_{3} \mathrm{OF}_{4} \cdot 2 \mathrm{HCl}$

1-[1-(4-Fluorophenyl)-1H-3-indolyl]-3-[4-(2-pyridyl)piperazin-1-yl]-1-propanone $2 \mathrm{i}$

mp $265^{\circ} \mathrm{C}$; Yield $62 \%$ (methanol-ether) IR $\left(\mathrm{cm}^{1}\right): 2475$ ( $\left.{ }^{+} \mathrm{NHCl}\right) ; 1640(\mathrm{C}=\mathrm{O}) ; 1590$ (aromatic); $1530,1180(\mathrm{C}-\mathrm{N}) ; 1545(\mathrm{C}=\mathrm{N})$. ${ }^{1} \mathrm{H}$ NMR (DMSO-d 6 ): 8.27-6.99 (m,13H,Ar-H); 3.93-3.27 (m,10H, $\left.\mathrm{CH}_{2}\right) ; 2.53\left(\mathrm{~s}, 2 \mathrm{H}, \mathrm{CH}_{2}\right)$. Anal. $\mathrm{C}_{25} \mathrm{H}_{25} \mathrm{~N}_{4} \mathrm{OF}, 2 \mathrm{HCl}$

1-[1-(4-Fluorophenyl)-1H-3-indolyl]-3-[4-(2-pyrimidinyl)piperazin-1-yl]-1-propanone 2j

$\mathrm{mp} 235^{\circ} \mathrm{C}$; Yield $61 \%$ (methanol-ether); IR $\left(\mathrm{cm}^{-1}\right): 2510$ ( $\left.{ }^{+\mathrm{NHCl}}\right) ; 1630(\mathrm{C}=\mathrm{O}) ; 1590$ (aromatic); 1530,1180 (C-N); 1565 (C=N). ${ }^{1} \mathrm{H}$ NMR (DMSO-d $)$ : 8.47-6.73 (m,12H,Ar-H) ; 4.00-3.15 (m, $\left.10 \mathrm{H}_{2} \mathrm{CH}_{2}\right) ; 2.65\left(\mathrm{~s}, 2 \mathrm{H}, \mathrm{CH}_{2}\right)$. Anal. $\mathrm{C}_{25} \mathrm{H}_{2+1} \mathrm{~N}_{3} \mathrm{OF} .2 \mathrm{HCl}$ 


\section{Pharmacology}

Literature reports suggest that the orientation of therapeutic activity for indoles substituted with a 4-fluorophenyl moiety at the indolyl nitrogen, predominantly are towards treatment of various psychotic disorders (11) depending on other substituents present on the molecule. The main receptors targeted for such types of activities are the dopamine $\left(\mathrm{D}_{1} / \mathrm{D}_{2}\right)$ and serotonin $\left(5-\mathrm{HT}_{2}\right)$ receptors.

\section{Evaluation of Apomorphine-induced Cage Climbing Behaviour}

Male albino mice were used. The effect of pretreatment with $30 \mathrm{mg} / \mathrm{kg}$ doses of the test compounds on apomorphine $(0.5 \mathrm{mg} / \mathrm{kg})$-induced cage climbing behaviour was studied by a literature method (12). Haloperidol, $1.0 \mathrm{mg} / \mathrm{kg}$ (i.p.) was used as control as it completely inhibited the climbing induced by apomorphine.

\section{Evaluation of Reversal of Haloperidol-induced Catalepsy [HIC]}

Male albino mice were tested for catalepsy by a literature method (13). Catalepsy was scored by a literature method (14). The test compounds $(30 \mathrm{mg} / \mathrm{kg}$ ) were injected 30 minutes before the injection of haloperidol $(0.25 \mathrm{mg} / \mathrm{kg})$. The catalepsy score was recorded at 30,60,120 and 180 minutes after haloperidol administration. The percentage reversal is calculated as a percentage of the difference between the total points obtained between control and test drugs.

\section{Results \& Discussion}

Ten novel arylpiperazinyl mannich bases of 1-(4-fluorophenyl)-3-acetyl indole have been synthesized. Mannich reaction of 1-(4-fluorophenyl)-3-acetyl indole with various arylpiperazines using paraformaldehyde in ethanol gave the arylpiperazinyl mannich bases in $46-62 \%$ yields. The lower yields are attributed to the large bulk of the N-4 unsubstituted arylpiperazines, which were used as the secondary amines for the mannich reaction in this study.

The target compounds were screened for dopaminergic $\left(D_{1} / D_{2}\right)$ receptor for antagonism of apomorphine induced climbing in mice in vivo. This screening method is predictive of potential antipsychotic activity since all clinically effective neuroleptics antagonize climbing and possess dopamine $\left(\mathrm{D}_{1} / \mathrm{D}_{2}\right)$ antagonist properties (15).

Of the compounds evaluated for their antagonism to dopamine $\left(D_{1} / D_{2}\right)$ receptors in the inhibition of apomorphine induced climbing behaviour in mice, compounds $\underline{\mathbf{2}} \mathbf{b}, \underline{\mathbf{2}} \mathbf{\mathrm { h }} \& \underline{\mathbf{2}}$ have shown higher antagonism, whereas compounds $\underline{2 a}, \underline{2 c}, \underline{2 f} \& \underline{2 i}$ on the other hand have shown lower antagonism (Table 1). All clinically effective antipsychotic agents act as antagonists at the dopaminergic $\left(D_{1} / D_{2}\right)$ receptor $(16)$ whose blockade is also associated with extrapyramidal side-effects (EPS). 
Induction of catalepsy is viewed as a compounds ability to cause EPS in man and antagonism of the serotonergic $\left(5-\mathrm{HT}_{2}\right)$ receptor has been reported to block or reverse the $\operatorname{HIC}(17,18)$.

These compounds were further evaluated for their arypicality in antipsychotic activity by their ability to reverse the catalepsy induced by haloperidol. Of these, only compounds $\underline{\mathbf{2}}, \underline{\mathbf{2}} \mathbf{b}, \underline{\mathbf{2}}$ \& $\underline{\mathbf{2}} \mathbf{i}$ showed minimal reversal of HIC indicating a probable lack of antagonism at serotonin $\left(5-\mathrm{HT}_{2}\right)$ receptors. (Table $\mathrm{l}$ )

Table 1. Results of 1-(4-fluorophenyl)- piperazinyl indolyl propanones [2a-j] for their inhibition of apomorphine induced climbing behaviour (A) and reversal of haloperidol induced catalepsy in mice (B).

\begin{tabular}{|c|c|c|c|}
\hline Compound No. & $\mathbf{R}$ & A & B \\
\hline $2 a$ & Phenyl & $36.4 \pm 6.3$ & $14.41 \pm 4.6$ \\
\hline $2 b$ & 3-Chlorophenyl & $58.3 \pm 5.9$ & $18.75 \pm 6.3$ \\
\hline $2 c$ & 4-Chlorophenyl & $25.4 \pm 6.3$ & $\neq$ \\
\hline $2 d$ & 4-Fluorophenyl & $\neq$ & \# \\
\hline $2 e$ & 2-Methoxyphenyl & $\neq$ & \# \\
\hline $2 f$ & 3-Methoxyphenyl & $24.6 \pm 7.9$ & $\neq$ \\
\hline $2 \mathrm{~g}$ & 4-Methoxyphenyl & $\neq$ & \# \\
\hline $2 \mathrm{~h}$ & 3-Trifluoromethyl phenyl & $69.5 \pm 8.5$ & $20.83 \pm 5.8$ \\
\hline $2 \mathrm{i}$ & 2-Pyridyl & $72.6 \pm 5.3$ & $33.33 \pm 5.7$ \\
\hline $2 \mathrm{j}$ & 2-Pyrimidinyl & $25.6 \pm 6.4$ & $\neq$ \\
\hline Haloperidol & - & 100 & Nil \\
\hline
\end{tabular}

$\neq$ - Not Significant. Inhibition less than 10\%; \# - Not Tested

A - \% Inhibition of apomorphine induced climbing (Mean \pm S.E.)

B - \% Reversal of haloperidol induced catalepsy (Mean \pm S.E.)

The $D_{1} / D_{2}$ antagonism exhibited provides a strong point towards the antipsychotic behaviour of these compounds.

Our approach was based on the scope offered by indolylalkylamines for structural modification towards effective CNS function. Although the amine moieties used were arylpiperazines, which are the basic structural templates with affinities for the 5-HT receptors, we observed that the compounds failed to antagonize $5-\mathrm{HT}_{2}$ receptors. Though a 4-fluorophenyl moiety on an indolyl system has been reported (11) for high affinity at the $5-\mathrm{HT}_{2}$ receptor, it should be noted that the presence of other pendant substituents on the indolyl system determines the specificity for a particular receptor subtype.

In another study, the replacement of the 4-fluorophenyl moiety with a 4-fluorobenzoyl moiety on the same indolyl system has been found to enhance the 5- $\mathrm{HT}_{2}$ antagonism along with the required dopamine antagonism providing good lead molecules for atypicality in antipsychotic activity (unpublished results). 
In view of the involvement of $D_{1} / D_{2}$ receptor antagonism (in inhibition of apomorphineinduced climbing behaviour) and possible role of $5-\mathrm{HT}_{2}$ antagonism (in haloperidolinduced catalepsy reversal), the present study needs further investigations to specify the structural features of these compounds for enhancement of their selectivity tor serotonergic and dopaminergic receptors and their subtypes for their modulatory action.

\section{Acknowledgement}

The financial assistance provided by the University Grants Commission, New Delhi is thankfully acknowledged.

\section{References \& Notes}

1. A. Mahamadi, P. Parimoo, W.G. Haney, B.F. Grabowski. J. Pharm. Sci., 62, (3) 490-492 (1973)

2. V. Erspamer, in "Progress in Drug Research", vol.3, E.Jucker, Ed., Interscience Publishers, Inc., New York, N.Y. (1961) p161, 329.

3. R.A. Glennon, P.K. Gessner. J. Med. Chem., 22, (4) 428-432 (1979)

4. J. Offermeier, E.J. Ariens. Arch. Int. Pharmacodyn. Ther., 164. 216 (1966)

5. H.H. Keasling, R.E. Willette, J. Szmuszkovicz. J. Med. Chem., 7, 94-96 (1964)

6. A.R.Subramanian,S.A.V.Raghavan, R. Jagadeesh Babu, C.N.V.H.B.Gupta, A.Veeranjaneyulu, P.Parimoo, P. Srinivas. Heterocyclic Communications (in press)

7. M. Tramontini, L.Angiolini. in "Mannich Bases: Chemistry and Uses," CRC Press Inc, Boca Raton, FL 1994

8. J.E. Saxton. J. Chem. Soc.,(P) 3592 (1952)

9. Data for 3-acetyl indole : prepared from indole using acetic anhydride and glacial acetic acid (9:1) to give 1,3-diacetylindole in overall $62 \%$ yield, followed by hydrolysis with aqueous $2 \mathrm{~N} \mathrm{NaOH}$ to give 3 -acetyl indole in $76 \%$ yield as long colorless prisms, m.p. $191^{\circ} \mathrm{C}$. IR $\left(\mathrm{cm}^{-1}\right)$ [KBr]: $3400(\mathrm{~N}-\mathrm{H}) ; 1650(\mathrm{C}=\mathrm{O}) ; 1605$ (aromatic);745(1,2-disubstitutedbenzene). 'HNMR(DMSO-d $\left.\mathrm{d}_{6}\right) ; \quad \delta \quad 7.99-6.81$ $(\mathrm{m}, 6 \mathrm{H}, \mathrm{Ar}-\mathrm{H}) ; \delta 2.20\left(\mathrm{~s}, 3 \mathrm{H}, \mathrm{CH}_{3}\right)$. Anal. $\mathrm{C}_{10} \mathrm{H}_{9} \mathrm{NO}$

10. J. Szmuszkovicz. J. Am. Chem. Soc., 82, 1180-1186 (1960)

11. J. Perregaard, J. Arnt, P.B. Klaus, J. Hyttel, C. Sanchez, C. J. Med. Chem., 35,1092 (1992). J. Perregaard, K. Andersen, J. Hyttel, C. Sanchez. J. Med. Chem., 35, 4813 (1992). J. Perregaard, J. Arnt, K. Andersen, J.B. Nielsen, M. Begtrup. J. Med. Chem., 35, 4823 (1992).

12. G. Costall, R.J. Naylor, V. Nohria. Eur. J. Pharmacol., 50, $39-50$ (1978)

13. B. Costall, R.J. Naylor. Eur. J. Pharmacol., 27, 46-58 (1974b)

14. P.A. Shore, R.L. Dorris. Eur. J. Pharmacol., 30, 315-318 (1975)

15. N.J.Hrib, J.G.Jurcak, F.P.Huger, C.L.Errico, R.W.Dunn. J. Med. Chem., 34, 1068-1072 (1991)

16. I. Casariego, C.F. Masaguer, E. Ravina. Tetrahedron Letters. 38, (31) 5555-5558 (1997)

17. P.B. Hicks. Life Sci., 47, (18) 1609-1615 (1990)

18. S. Kapur. Psychopharmacology. 124, 35-39 (1996) 